\title{
Variabilidad en la abundancia y retención de larvas de crustáceos decápodos en el estuario de Bahía Blanca, Provincia de Buenos Aires, Argentina
}

\author{
Patricia M. Cervellini \\ Departamento de Biología, Bioquímica y Farmacia \\ Universidad Nacional del Sur, San Juan 670, 8000 Bahía Blanca \\ E-mail: pcervell@criba.edu.ar
}

Recibido: 10 mayo 1999; versión corregida: 12 junio 2001; aceptado: 20 septiembre 2001

\begin{abstract}
RESUMEN. Se estudió la distribución de larvas de crustáceos decápodos dentro del estuario de Bahía Blanca con el objeto de comprobar la hipótesis de que algunas especies pueden retenerse en el estuario y otras son dispersadas aguas afuera pudiendo reinvadirlo. Se identificaron larvas de 30 especies, encontrándose las mayores abundancias de zoeas de Chasmagnathus granulata (84\%) y Cyrtograpsus altimanus (60\%). Un gran número de especímenes de estas dos especies se observó entre $120 \mathrm{~km}$ y $80 \mathrm{~km}$ en la zona interna del estuario con valores de $6.000 \mathrm{ind} / 100 \mathrm{~m}$; la máxima densidad se encontró a $110 \mathrm{~km}(12.441$ ind/100 m³) hallándose las cuatro zoeas de $C h$. granulata y cinco de $C$. altimanus. En la boca, las densidades de estas dos especies disminuyeron notablemente. Esta misma tendencia se observó en zoeas de Majidae y Xanthidae. Las zoeas de Pagurus exilis, Pinnotheres maculatus, Pinnixa patagoniensis y Corystoides chilensis fueron poco abundantes y sus densidades se incrementaron en la boca del estuario. Palabras claves: larvas, decápodos, crustáceos, estuario, Bahía Blanca, Argentina.
\end{abstract}

\section{Abundance and retention variability of Decapod Crustacea larvae in Bahía Blanca Estuary (Provincia de Buenos Aires, Argentina)}

\begin{abstract}
Distribution of larvae of decapod crustacean was studied in the Bahía Blanca estuary with the objective of testing the hypothesis that some species can be retained in the estuary and other can be dispersal offshore waters and can be reinvaded. Larvae of 30 species were identificated, the most abundance corresponding to Chasmagnathus granulata (84\%) and Cyrtograpsus altimanus (60\%) respectively. A high number of stages of these species were found between $120 \mathrm{~km}$ and $80 \mathrm{~km}$ in the inner zone of the estuary with $6000 \mathrm{ind} / 100 \mathrm{~m}^{3}$. The maximum density was found at $110 \mathrm{~km}\left(12.441 \mathrm{ind} / 100 \mathrm{~m}^{3}\right)$. And here was found life cycle of Ch. granulata and C. altimanus. In the mouth of estuary, the density of both species decayed. The same tendency was observed to Majidae and Xanthidae zoeas. Pagurus exilis, Pinnotheres maculatus, Pinnixa patagoniensis and Corystoides chilensis were less abundant but their densities were incremented offshore waters.
\end{abstract}

Key words: larvae, decapod, crustaceans, estuary, Bahía Blanca, Argentina.

\section{INTRODUCCIÓN}

Los patrones de distribución que tienen algunas larvas de crustáceos decápodos dentro de sistemas estuariales y en zonas adyacentes a bahías, sugieren medios por los cuales los jóvenes estadios pueden reclutarse a las poblaciones de adultos bentónicos y bentopelágicos (Sandifer, 1975; Scheltemea, 1975; Lambert y Epifanio, 1982, McConaugha, 1988). El reclutamiento de las poblaciones de estos invertebrados marinos requiere suficiente número de larvas o juveniles que sobrevivan y permanezcan dentro del estuario, ya que pueden ser llevados hacia la boca del mismo o retornar a la región en diferentes estadios de su ciclo de vida (postlarva, megalopa, juvenil), incorporándose nuevamente al stock 
parental (Dittel y Epifanio, 1982).

Sin embargo, la mayoría de las larvas de decápodos pelágicos o bentopelágicos están a merced de las corrientes en su desplazamiento horizontal. Como en la mayor parte de los estuarios, el flujo neto es hacia el mar, estos organismos corren el riesgo de ser dispersados (Sandifer, 1975; Epifanio, 1988). La distancia que las larvas pelágicas pueden desplazarse de sus poblaciones en el estuario y a la inversa, la oportunidad que tienen de retenerse dentro, dependerá de la interacción de una serie de factores, como la magnitud de la marea (transporte neto), distribución de las larvas respecto a la profundidad, tolerancia y retención a cambios de las condiciones ambientales (salinidad, temperatura, oxígeno) y la duración de la vida planctónica.

En el presente trabajo se ha cuantificado la abundancia de larvas de crustáceos decápodos en el estuario de Bahía Blanca, para contrastar la hipótesis que las larvas de algunas especies pueden retenerse en el estuario, modificando de este modo sus abundancias, mientras otras son dispersadas, pudiendo reinvadir nuevamente el estuario en diferentes estadios de su ciclo de vida.

\section{MATERIALES Y MÉTODOS}

Se recolectaron 52 muestras de plancton en seis estaciones ubicadas a lo largo del canal principal de navegación del estuario de Bahía Blanca (Fig. 1). Las salidas mensuales se efectuaron con el guardacostas GC 75 de la Prefectura Naval Argentina, a excepción de marzo y julio que por inconvenientes climáticos no se llegó a destino. Estas correspondieron a la Campaña Canal I (septiembre 1982 agosto 1983) que cubrió la boca del estuario. Las larvas se capturaron con red Hensen de $50 \mathrm{~cm}$ de diámetro de boca, 1,90 m de largo y $224 \mathrm{~mm}$ de apertura de poro. Se efectuaron arrastres horizontales superficiales y oblicuos de 10 minutos de duración a 2 nudos y las muestras se fijaron con formaldehído al 4\%. Se utilizó un flujómetro para estimar el volumen filtrado por la red y el número de larvas fue convertido a ind $/ 100 \mathrm{~m}^{3}$. Se indicó la abundancia promedio para cada una de las especies de Decapoda en las diferentes estaciones del muestreo.

Los resultados de distribución espacial de las larvas se compararon con los datos de 240 muestras de plancton colectadas en siete estaciones de muestreo efectuadas durante

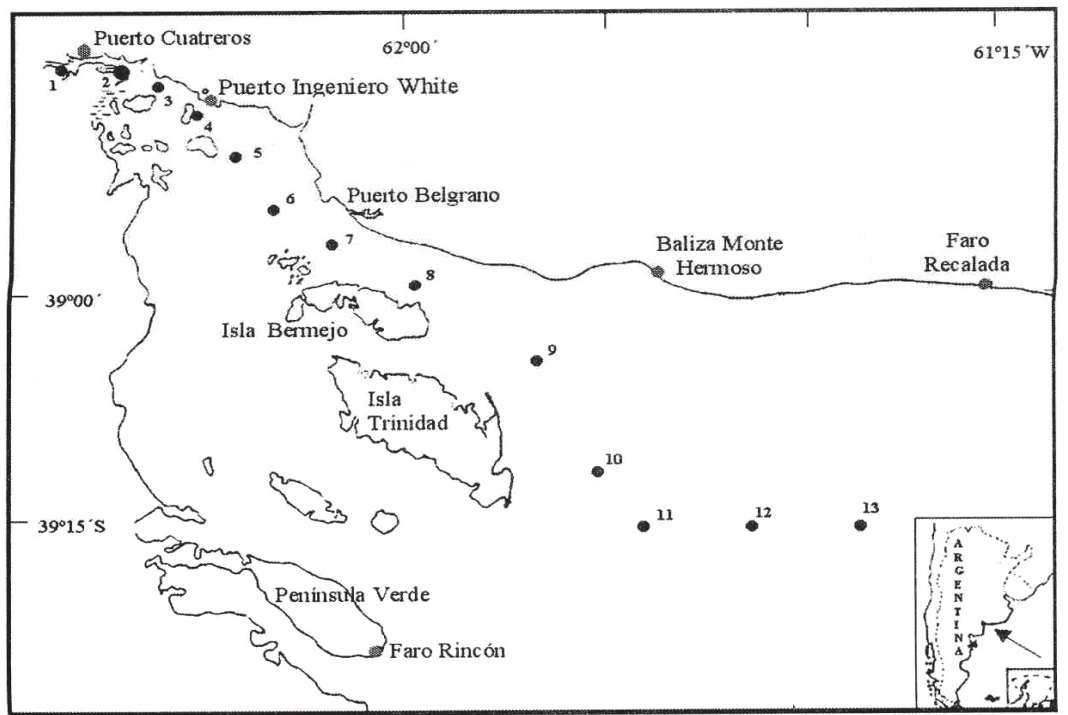

Figura 1. Mapa del estuario de Bahía Blanca, localización geográfica y ubicación de las estaciones de muestreo.

Figure 1. Map of the Bahía Blanca estuary, showing station locations. la Campaña Canal II (septiembre 1982 - noviembre 1985) que abarcó la porción interna de estuario (Cervellini,1992). Para establecer la significancia estadística de los máximos de abundancia en las diferentes zonas de muestreo, se realizó un análisis de varianza, previa verificación del requisito de homocedasticidad (Test de Barlett). Adicionalmente, se midió la temperatura con termómetro de superficie. Las determinaciones de salinidad y oxígeno disuelto se efectuaron al día siguiente del muestreo mediante un salinómetro de inducción Beckman RS7-B y el método de Winkler modificado según Carrit y Carpenter (1966) respectivamente. La transparencia del agua se midió con el disco Secchi. 


\section{RESULTADOS}

Se identificaron los estadios larvales de 30 especies de Decapoda y para su identificación se siguieron las claves de (Boschi et al., 1992). A partir de septiembre de 1982 las larvas de decápodos comenzaron a reclutarse en la boca del estuario. La distribución de abundancia para el total de especies capturadas evidenció una marcada variación estacional (Fig. 2).

Durante el período de muestreo se observaron valores más altos en octubre, noviembre y diciembre, siendo el máximo en enero de 1983 con 9.092 ind/100 $\mathrm{m}^{3}$, el reclutamiento continuó en verano hasta su declinación a partir de abril con 88 ind $/ 100 \mathrm{~m}^{3}$. De las 30 especies identificadas, las zoeas de Ch. granulata y C. altimanus fueron muy importantes representando el $84 \%$ y $60 \%$ de la población en primavera y verano respectivamente (Fig. $3)$. De estas dos especies se colectaron todos sus estadios larvales, aunque esporádicamente se encontraron algunas megalopas, lo que se debería a la mezcla turbulenta en la zona de captura y al tipo de arrastre efectuado. El segundo lugar estuvo representado por zoeas de Pagurus exilis (7\%) y Pachycheles haigae (19\%). Las restantes especies fueron escasas y su abundancia no superó el 4\% (Tabla 1).

Comparando los resultados de ambas campañas (Canal I y Canal II) se analizaron las densidades de larvas de Ch. granulata y C. altimanus para las 13 estaciones de muestreo. Estas variaciones se esquematizaron en relación con la distancia desde la boca del estuario (Fig. 4). Un alto número de estadios se colectó entre $120 \mathrm{~km}$ y $80 \mathrm{~km}$, siempre con densidades superiores a $6.000 \mathrm{ind} / 100 \mathrm{~m}^{3}$. La máxima densidad se encontró a $110 \mathrm{~km}$ en la parte interna del estuario (12.441 ind/100 $\left.\mathrm{m}^{3}\right)$, hallándose las cuatro zoeas y megalopas de Ch. granulata, y las cinco zoeas y megalopas de $C$. altimanus. Cerca de de la boca del estuario, las abundan-

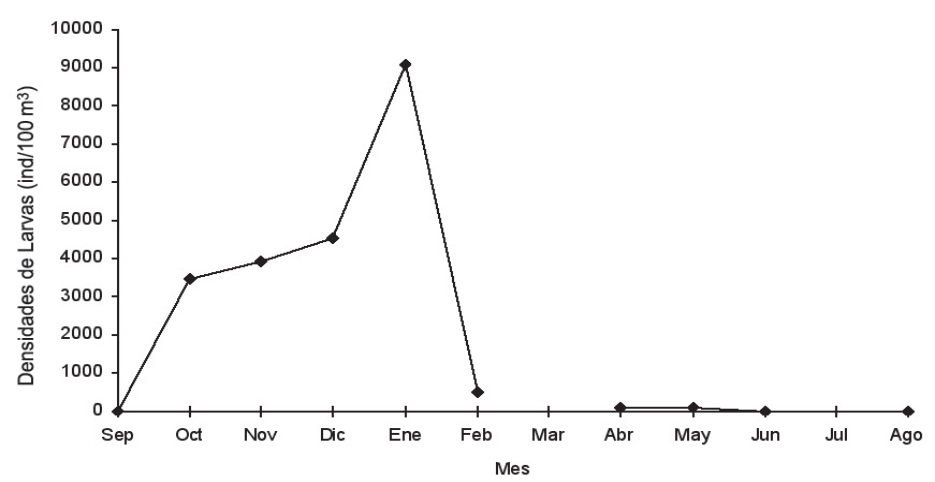

Figura 2. Abundancia media del total de larvas de Decapoda (ind/ $100 \mathrm{~m}^{3}$ ) durante el período septiembre 1983 - agosto 1985.

Figure 2. Mean total abundance (ind $/ 100 \mathrm{~m}^{3}$ ) of decapod crustaceans during the period September 1982 - Ausgust 1985.

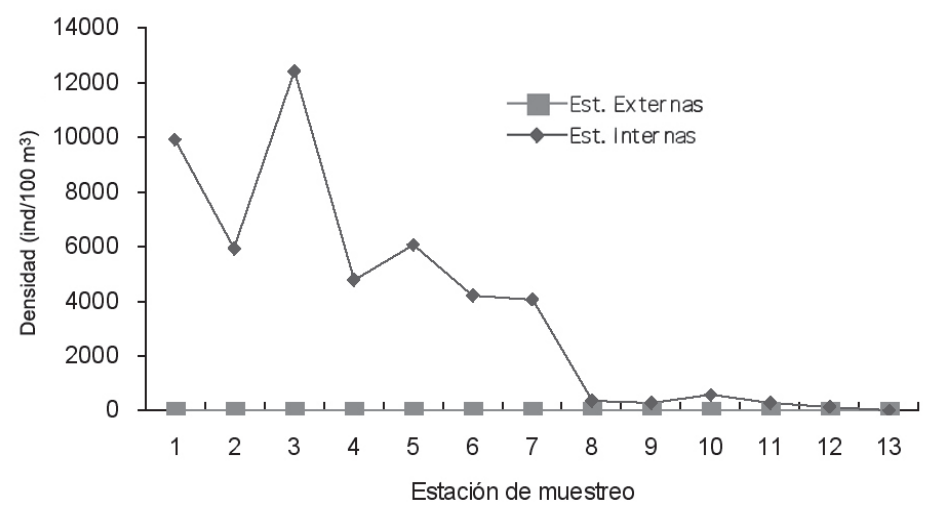

Figura 3. Distribución espacial de zoeas de Chasmagnathus granulata y Cyrtograpsus altimanus.

Figure 3. Spatial distribution of zoeal stages of Chasmagnathus granulata and Cyrtograpsus altimanus.

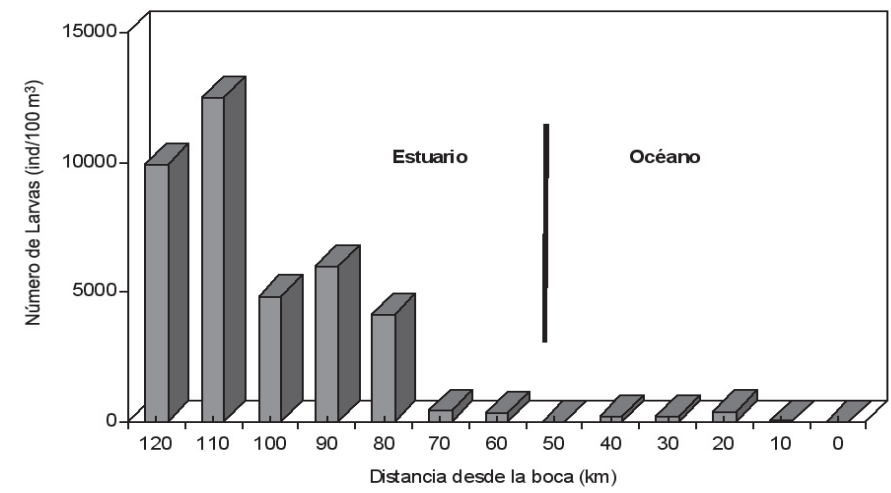

Figura 4. Cambios en la densidad larval de Chasmagnathus granulata y Cyrtograpsus altimanus, en relación a la distancia desde la boca del estuario (agosto 1982 - noviembre 1985).

Figure 4. Changes in larval density of Chasmagnatus granulata and Cyrtograpsus altimanus with distance from the mouth of Bahia Blanca estuary (August 1982 - November 1985). 
Tabla 1. Lista de especies de crustáceos decápodos presentes en el estuario de Bahía Blanca.

Table 1. Decapod crustacean species present in Bahía Blanca estuary.

\begin{tabular}{|c|c|c|c|}
\hline Suborden & Infraorden & Familia & Especie \\
\hline \multirow[t]{3}{*}{ Dendrobranchiata } & & Penaeidae & Artemesia longinaris \\
\hline & & Solenoceridae & Pleoticus muelleri \\
\hline & & Sergestidae & Peisos petrunkevitchi \\
\hline \multirow[t]{27}{*}{ Pleocyemata } & Caridea & Hippolytidae & Latreutes parvulus \\
\hline & & Alpheidae & Betaeus lilianae \\
\hline & & & Alpheus puapeba \\
\hline & Thalassinidea & Callianassidae & Callianassa $s p$ \\
\hline & Anomura & Porcellanidae & Pachycheles haigae \\
\hline & & & Pachycheles chubutensis \\
\hline & & Paguridae & Pagurus exilis \\
\hline & & & Paguristes sp \\
\hline & & & Loxopagurus loxochelis \\
\hline & & Albuneidae & Blepharipoda doelloi \\
\hline & Brachyura & Grapsidae & Chasmagnathus granulata \\
\hline & & & Cyrtograpsus altimanus \\
\hline & & & Cyrtograpsus angulatus \\
\hline & & Pinnotheridae & Pinnotheres maculatus \\
\hline & & & Pinnixa patagoniensis \\
\hline & & Xanthidae & Platyxanthus patagonicus \\
\hline & & & Platyxanthus crenulatus \\
\hline & & & Pilumnus reticulatus \\
\hline & & Portunidae & Ovalipes trimaculatus \\
\hline & & & Callinectes sapidus \\
\hline & & & Coenophtlamus tridentatus \\
\hline & & Atelecyclidae & Corystoides chilensis \\
\hline & & Majidae & Libinia spinosa \\
\hline & & & Pelia rotunda \\
\hline & & & Collodes rostratus \\
\hline & & & Leucippa pentagona \\
\hline & & & Rochinia gracilipes \\
\hline
\end{tabular}

cias disminuyeron notablemente, a $20 \mathrm{~km} 383$ ind/ $100 \mathrm{~m}^{3} \mathrm{y}$ aguas afuera $(10 \mathrm{~km})$ con $17 \mathrm{ind} / 100 \mathrm{~m}^{3}$.

Para observar diferencias significativas en la abundancia de larvas entre las distintas estaciones de muestreo, se realizó un análisis ANOVA entre estaciones, exceptuando la estación 5 por su proximidad a la 4 (Tablas 2 a 4), determinándose las siguientes agrupaciones:

Zona 1 = zona interna (estaciones 1-2-3-4)

Zona 2 = zona intermedia (estaciones 6-7-8-9)

Zona 3 = zona externa (estaciones 10-11-12-13)

De los resultados de las comparaciones entre las medias poblacionales de las tres zonas, se deduce que las medias entre las zonas 1 y 2 no se suponen distintas, mientras que la zona 3 tiene una densidad media muy diferente de las otras dos.

El Xanthidae P. reticulatus presentó la misma tendencia, con densidades de $56-58 \mathrm{nd} / \mathrm{IUU} \mathrm{m}^{3}$ al interior del estuario y $1,36 \mathrm{ind} / 100 \mathrm{~m}^{3}$ en la boca. (Fig. 5). Las otras dos especies de xántidos presentaron distribuciones espaciales diferentes. $P$. crenulatus se colectó en estaciones intermedias con altas densidades, en cambio las larvas de $P$. patagonicus se presentaron en una estación del año bien marcada y en bajas densidades (Fig. 6). Los Majidae presentaron un aumento en las estaciones 4 a 9 , con densidades que oscilaron de 6 a 100 ind/ $100 \mathrm{~m}^{3}$, disminuyendo gradualmente hacia la boca del estuario y aguas adyacentes. De las larvas capturadas, la especie más abundante fue Pelia rotunda (Fig. 7).

A continuación se detallan las larvas registradas únicamente fuera del estuario. Entre los Anomura, la más abundante fue Pagurus exilis (1580 ind/100 $\mathrm{m}^{3}$ ), seguida de Paguristes sp. con 444,3 ind/100 
Tabla 2. Resumen de datos muestrales transformados $(\log$ $($ densidad + 1)).

Table 2. Summary of transformed $(\log ($ density +1$))$ sample data.

\begin{tabular}{|cccc|}
\hline Zona & Media & $\begin{array}{c}\text { Desviación } \\
\text { estándar }\end{array}$ & Frecuencia \\
\hline 1 & 0,851 & 0,344 & 16 \\
2 & 0,632 & 0,347 & 16 \\
3 & 0,101 & 0,220 & 16 \\
TOTAL & 0,528 & 0,439 & 48 \\
\hline
\end{tabular}

Tabla 4. Comparación por zona (Scheffe) $\log$ $($ densidad +1$)$.

Table 4. Comparison by area (Scheffe) $\log$ (density + 1).

\begin{tabular}{ccc|}
\hline $\begin{array}{c}\text { Media de filas } \\
\text { Media de columnas }\end{array}$ & $\mathbf{1}$ & $\mathbf{2}$ \\
\hline 2 & $-0,219$ & \\
3 & $-0,750^{*}$ & $-0,531 *$ \\
\hline
\end{tabular}
* valores significativos $(\mathrm{P}<0,001)$

Tabla 3. Análisis de varianza de la abundancia de larvas entre las estaciones de muestreo.

Table 3. Larvae abundance between stations ANOVA.

\begin{tabular}{|lccccc|}
\hline Fuente & SS & g.l. & MS & F & Prob \\
\hline Entre & 4,76342391 & 2 & 2,38171195 & 24,88 & 0,000 \\
Dentro & 4,30782001 & 45 & 0,09572933 & & \\
TOTAL & 9,07124391 & 47 & 0,19300519 & & \\
\hline
\end{tabular}

Test de Bartlett; $\mathrm{Chi}^{2}$ (2 g.1.) $=3,4858$ prob $>\mathrm{Chi}^{2}=0,175$

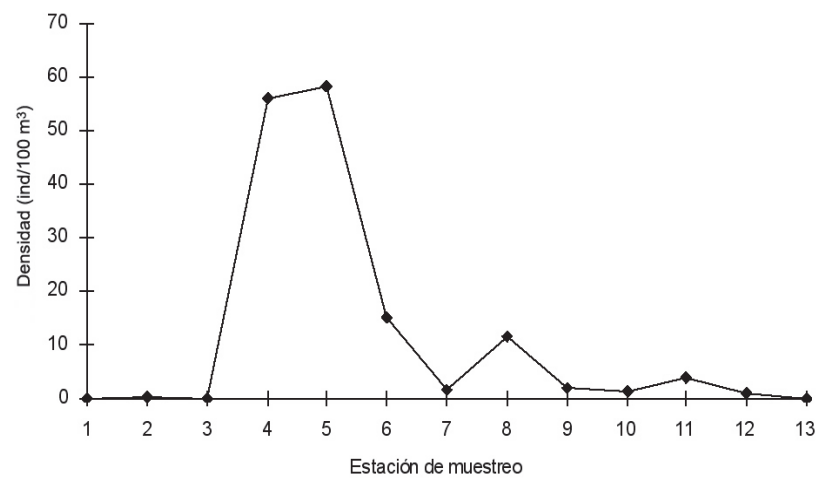

Figura 5. Distribución espacial de larvas de Pilumnus reticulatus.

Figure 5. Spatial distribution of Pilumnus reticulatus larvae.

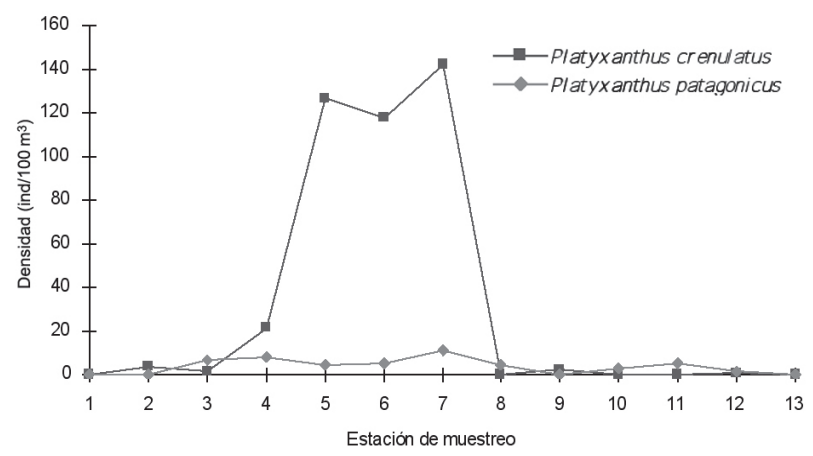

Figura 6. Distribución espacial de larvas de Platyxanthus patagonicus y Platyxanthus crenulatus.

Figure 6. Spatial distribution of Platyxanthus patagonicus and Platyxanthus crenulatus larvae.
Tabla 5. Cambios en la densidad de los estadios larvales de Chasmagnathus granulata y Cyrtograpsus altimanus en función de la distancia desde la boca del estuario de Bahía Blanca. MA: muy abundante, A: abundante, E: escaso y (-) ausente.

Table 5 Changes in density of larval stages of Chasmagnathus granulata and Cyrtograpsus altimanus as a function of distance from the mouth of Bahía Blanca estuary. MA: very abundant, A: abundant, E: low and (-) absent.

\begin{tabular}{|c|c|c|}
\hline & $\begin{array}{c}\text { Estuario } \\
\text { distancia }(\mathrm{km})\end{array}$ & $\begin{array}{c}\text { Océano } \\
\text { distancia }(\mathrm{km})\end{array}$ \\
\hline Estadio & $12010080 \quad 60$ & 40200 \\
\hline Zoea I & MA A MA A & A A - \\
\hline Zoea II & A $\quad$ A $\quad$ E $\quad$ E & A A - \\
\hline Zoea III & A A A & $\mathrm{E}$ \\
\hline Zoea IV & MA A A E & $\mathrm{E} \quad \mathrm{E}$ - \\
\hline Zoea V & MA A E & E E - \\
\hline Megalopa & MA A E & - \\
\hline
\end{tabular}


$\mathrm{m}^{3}$ y Loxopagurus loxochelis (Fig. 8). Pachycheles haigae se concentró en la boca del estuario (estaciones 9 y 10), con escasos estadios en la parte interna (Fig. 9).

Las zoeas de camarones Caridea y Penaeoidea se registraron por primera vez en mar abierto (estaciones 11,12 y 13) y en muy bajas densidades (Fig. 10). Un número reducido de diferentes especies apareció en forma irregular en el plancton, como Corystoides chilensis, Pinnixa patagoniensis y Pinnotheres maculatus, todas las cuales se capturaron en la boca del estuario (Fig. 11).

A continuación, se indica la abundancia relativa por estadios, de las dos especies de Grapsidae capturados en el área de estudio, de los cuales se pudo completar su ciclo de vida a partir de sus estadios planctónicos (Tabla 5).

\section{DISCUSIÓN}

Varios autores, como Sandifer (1973, 1975) y Dittel y Epifanio (1982), han encontrado larvas de especies comunes de decápodos mucho más abundantes en muestras de fondo que cerca de la superficie y han especulado que la tendencia a congregarse cerca del fondo, donde el transporte neto es contra la corriente, debería ser un mecanismo por el cual las larvas puedan retenerse dentro del estuario y subsecuentemente reclutarse en las poblaciones de adultos. Similares hipótesis han sido señaladas para otros zooplancteres estuariales y costeros (Cronin y Forward,1979; Wood y Argis, 1971).

En la actualidad, existen dos escuelas del pensamiento concernientes a los factores que controlan el transporte de larvas de invertebrados (Epifanio, 1988). La primera de ellas propone que los movimientos horizontales son controlados por factores físicos y la natación juega un peque-

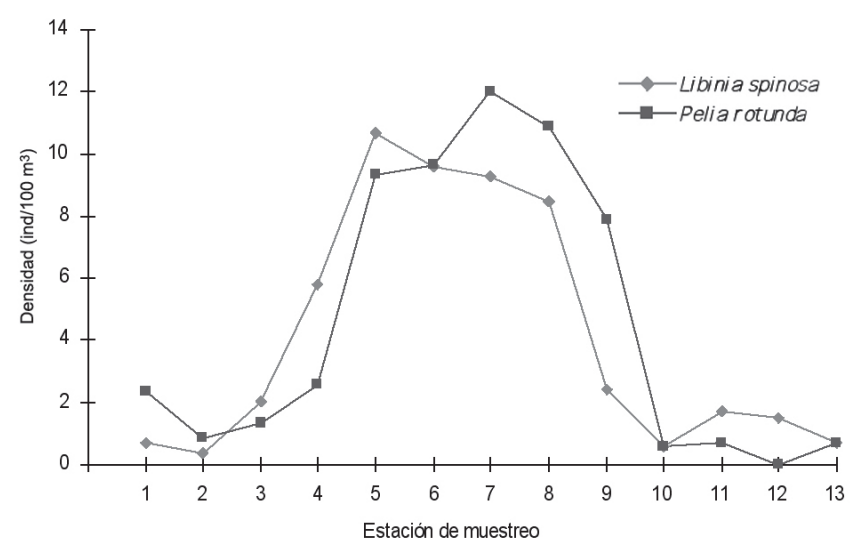

Figura 7. Distribución espacial de larvas de Majidae.

Figure 7. Spatial distribution of Majidae larvae.

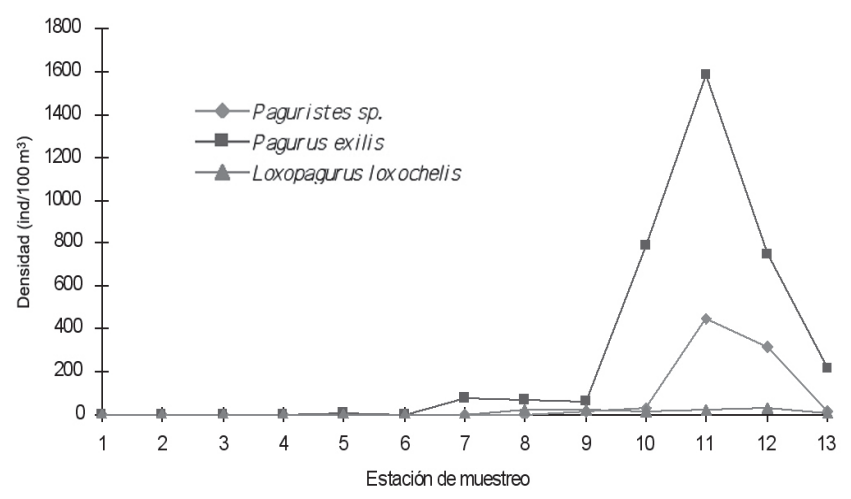

Figura 8. Distribución espacial de larvas de Anomura.

Figure 8. Spatial distribution of Anomura larvae.

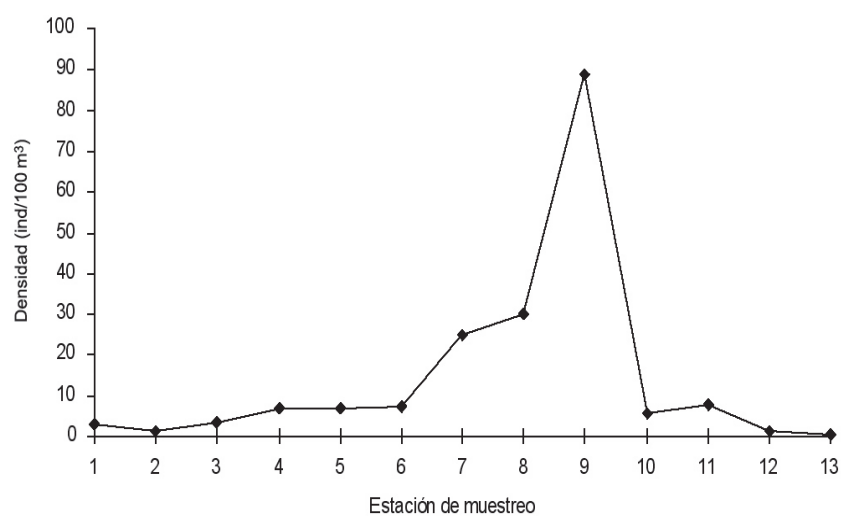

Figura 9. Distribución espacial de larvas de Pachycheles haigae. Figure 9. Spatial distribution of Pachycheles haigae larvae. 


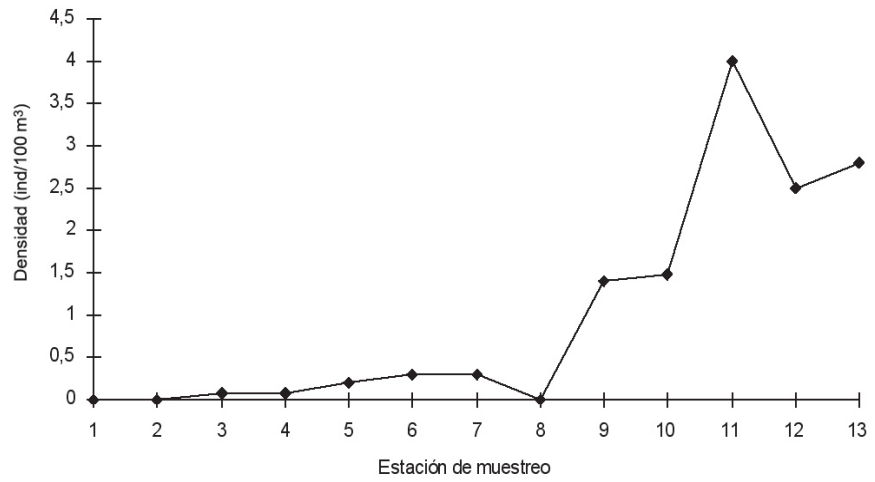

Figura 10. Distribución espacial de larvas de camarones Caridea y Penaeoidea.

Figure 10. Spatial distribution of shrimps Caridea y Penaeoidea larvae.

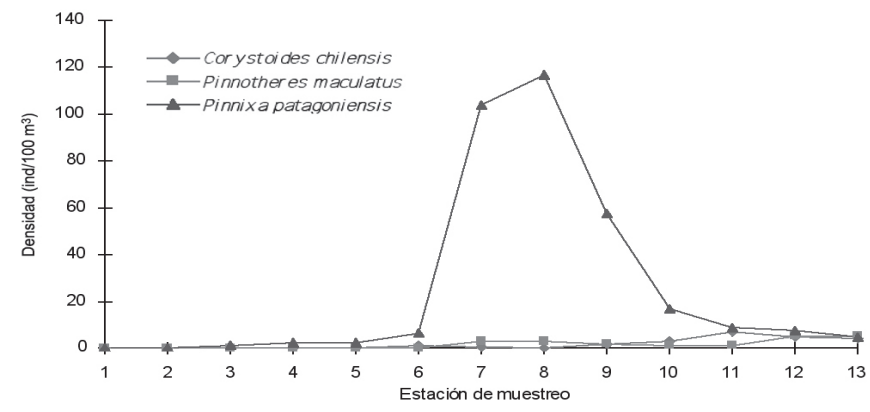

Figura 11. Distribución espacial de larvas de Corystoides chilensis, Pinnotheres maculatus y Pinnixa patagoniensis.

Figure 11. Spatial distribution of Corystoides chilensis, Pinnotheres maculatus and Pinnixa patagoniensis larvae.

ño rol en el proceso (hipótesis pasiva) (Boicourt, 1982; Seliger et al.,1982). Las larvas son diminutas y el efecto de su natación sostenida es insignificante comparado con la advección producida por el movimiento de marea y submarea (McConaugha, 1988).

La segunda que se refiere a una variación activa de este transporte para la retención de las larvas en el estuario (hipótesis activa), involucraría cambios en la distribución vertical como un proceso de desarrollo ontogenético (Bonsfield, 1955). Numerosos trabajos de laboratorio muestran que los estadios tempranos mantienen su posición en la parte superior de la columna de agua y así son transportados corriente abajo, mientras los últimos estadios larvales tienen una posición en la parte baja de la columna siendo llevados aguas arriba, afectando la retención en el estuario.
Las larvas en el estuario de Bahía Blanca fueron muy abundantes durante el período estival con una amplia variedad de formas; este ciclo anual ya ha sido observado por (Cervellini, 1988) para los años 1983 a 1987 y coincide con los datos de Hoffmeyer (1994) que estudió la sucesión anual del zooplancton en el estuario de Bahía Blanca. La tendencia en la fluctuación anual estuvo marcada por las zoeas de Chasmagnathus granulata y Cyrtograpsus altimanus, organismos que conforman los extensos cangrejales del área en cuestión, representando casi el $80 \%$ de los Decapoda. Las mayores abundancias observadas al interior del estuario (12.441 ind $\left./ 100 \mathrm{~m}^{3}\right)$ estarían indicando una posible retención en el estuario como lo señala (McConaugha, 1988). Lo mismo sucedería con el xánthido Pilumnus reticulatus. Esto sería una estrategia diferente a lo que ocurre en ambientes costeros, como la laguna de Mar Chiquita donde existe exportación de larvas de grápsidos que al no soportar cambios en los factores ambientales (bajas salinidades) son llevadas mar afuera (Anger et al.,1994). El factor condicionante de la salinidad en la porción interna del estuario de Bahía Blanca es la magnitud de las lluvias en la zona. Por su comportamiento, el estuario se puede considerar "verticalmente homogéneo" y en veranos muy calurosos donde la evaporación juega un papel muy importante se transforma en hipersalino (Freije et al.,1981; Piccolo y Perillo, 1990).

La abundancia de larvas de Platyxanthus patagonicus, Platyxanthus crenulatus y zoeas de la Familia Majidae, que presentaron densidades significativas en estaciones intermedias, coinciden con la presencia de adultos provenientes de los canales que conforman el estuario de Bahía Blanca (Elías, 1985).

La distribución de las zoeas de Paguristes sp, Pagurus exilis, Loxopagurus loxochelis y Pachycheles 
haigae que se registraron únicamente en la boca, es coincidente con la de los adultos en el bentos (Bremec y Cazzaniga, 1984; Bremec, 1986).

Es sabido que los estadios larvales, en particular de camarones Peneidos, requieren de condiciones de mar estables, pues pequeñas variaciones afectan la supervivencia de las larvas (García y Le Reste, 1987). Al respecto, las larvas y postlarvas de los camarones Caridea y Penaeoidea se registraron solamente en la desembocadura donde los factores abióticos, fundamentalmente la salinidad, son más constantes asemejándose a los de la plataforma continental adyacente (Piccolo y Perillo, 1997).

Con relación a la abundancia de larvas por estadio, los resultados indicaron para los Grapsidae, un número importante de zoeas I, II, III y IV en la parte superior de la columna de agua y en las estaciones internas de muestreo, siendo escasos los estadios tempranos de desarrollo capturados en la boca del estuario. Las megalopas fueron más abundantes en muestras de fondo, indicando su posición en la parte inferior de la columna de agua, afectando así la retención de las dos especies determinadas.

\section{REFERENCIAS}

Anger, K.E. 1994. Hatching rhythms and dispersion of decapod crustacean larvae in a brackish coastal lagoon in Argentina. Helgolander Meeresunters, 48: 445-466.

Boicourt, W.C. 1982. Estuarine larval retention mechanisms on two scales. En: V. Kennedy (ed). Estuarine comparisons. Academic Press, New York, pp 445-466.

Bonsfield, E.L. 1955. Ecological control of ocurrence of barnacles in the Miramichi estuary. Bull. Nat. Mus. Can.Biol. Ser., 137: 1-65.

Boschi E.E., E.C. Fischbach y M.I. Iorio. 1992. Catálogo ilustrado de los crustáceos estomatópodos y decápodos marinos de Argentina. Frente Marítimo, 10A: 7-94.

Bremec, C. 1986. Inventario y ecología del macrobentos marino de un sector de la Provincia Bonaerense. Tesis doctoral Universidad Nacional de La Plata, 209 pp.

Bremec, C. y N. Cazzaniga.1984. Consideraciones sobre Pachycheles haigae Rodrigues da Costa,1960 y Pachycheles chubutensis Boschi, 1963 en Monte Hermoso, Argentina (Crustacea, Anomura, Porcellanidae). Iheringia, Ser. Zool., 64: 149-162.

Carritt, D.E. y J.H. Carpenter. 1966. Comparison and evaluation of currently employed modifications of the Winkler method for determining dissolved oxygen in seawater; a NASCO Report. J. Mar. Res., 24: 286-318.

Cervellini, P.M. 1988. Las larvas y postlarvas de los crustáceos Decapoda en el estuario de Bahía Blanca. Variaciones estacionales y su relación con los factores ambientales. Tesis Doctoral, Universidad Nacional del Sur, Bahía Blanca, Argentina, 246 pp.

Cervellini, P.M. 1992. Seasonal and spatial distribution of decapod Crustacea larvae in the Bahía Blanca estuary, Argentina. J. Aqua. Trop., 7: 35-46.

Cronin, T.W. y R.B. Forward. 1979. Tidal vertical migration: an endogenous rhythm in estuarine crab larvae. Science, 204: 1020-1022.

Dittel, A.I. y C.E. Epifanio. 1982. Seasonal abundance and distribution of crab larvae in Delaware Bay. Estuaries, 5: 197-202.

Elías, R. 1985. Macrobentos del estuario de Bahía Blanca (Argentina ) I. Mesolitoral. Spheniscus, 3: 51-52.

Epifanio, C.E. 1988. Transport of invertebrate larvae between estuaries and the continental shelf. Amer. Fish. Soc. Symp., 3: 104-114.

Freije, R.H., R.O. Asteasuian, A. Schmitdt y J.R. Zavatti. 1981. Relación de la salinidad y temperatura del agua con las condiciones hidrometeorológicas en la porción interna del estuario de Bahía Blanca. Contribución Científica del Instituto Argentino de Oceanografía, 57: 1-18.

García, S. y L. Le Reste.1987. Ciclos vitales, dinámica, explotación y ordenación de las poblaciones de camarones peneidos costeros. FAO, Doc. Téc., 203: $1-180$.

Hoffmeyer, M.S. 1994. Seasonal succesion of Copepoda in the Bahía Blanca estuary. Hydrobiol., 292/293: 303-308.

Lambert, R. y C.E. Epifanio. 1982. A comparison of dispersal strategies in two genera of brachyuran crabs in a secondary estuary. Estuaries, 5: 182-188. 
McConaugha, J.R. 1988. Export and reinvasion of larvae as regulators of estuarine Decapod populations. Amer. Fish. Soc. Symp., 3: 90-103.

Piccolo, M.C. y M.E. Perillo. 1990. Physical characteristics of the Bahía Blanca estuary (Argentina). Estuar. Coast. Shelf Sci., 31: 303-317.

Piccolo, M.C. y M.E. Perillo 1997. Geomorfología e hidrografía de los estuarios. En: E.E. Boschi (ed.). El Mar Argentino y sus Recursos pesqueros. Tomo 1. Instituto Nacional de Investigación y Desarrollo Pesquero, Mar del Plata. Argentina, pp. 133-161.

Sandifer, P.A. 1973. The role of pelagic larvae in recruitment to populations of adults decapod crustaceans in the York River estuary and adjacent lower Chesapeake Bay, Virginia, 1968-1969. Estuar. Coast. Mar. Sci., 14: 235-257.
Sandifer, P.A. 1975. The role of pelagic larvae in the recruitment to populations of adults decapod crustacean in the York River estaury and adjacent lower Chesapeake Bay, Virginia, Estuar. Coast. Mar. Sci., 3: 269-279.

Scheltemea, R.S. 1975. Relationship of larval dispersal gene flow and natural selection to geographic variation of benthic invertebrates in estuarine and along coastal regions. Estuar. Res., 1: 372-391.

Seliger, H.H., J.A. Boggs, R.B. Rivkin, W.H. Bigley y K.R. Aspden.1982. The transport of oyster larvae in estuary. Mar. Biol., 71: 57-72.

Wood, L. y W.J. Hargis. 1971. Transport of bivalve larvae in a tidal estuary. En: D. Crisp (ed.). Fourth European Marine Biology Symposium, Cambridge University Press, Cambridge, pp. 29-44. 